\title{
BMJ Open Quality Increasing patient flow through neurosurgical critical care: the Leeds Improvement Method
}

\author{
James Meacock (D), Soumya Mukherjee, Asim Sheikh
}

To cite: Meacock J, Mukherjee S, Sheikh A. Increasing patient flow through neurosurgical critical care: the Leeds Improvement Method. BMJ Open Quality 2021;10:e001143. doi:10.1136/ bmjoq-2020-001143

Received 5 August 2020 Accepted 15 May 2021

Check for updates

(C) Author(s) (or their employer(s)) 2021. Re-use permitted under CC BY-NC. No commercial re-use. See rights and permissions. Published by BMJ.

Neurosciences, Leeds Teaching Hospitals NHS Trust, Leeds, UK

Correspondence to

Dr James Meacock;

James.Meacock@nhs.net

\section{ABSTRACT}

At Leeds General Infirmary, a busy tertiary centre for neurosurgery, there has been little visibility of the stepdown status of the patients from intensive care to high dependency or from the latter to a ward bed. The only record of the current situation was limited to the paper notes of the bed managers. Furthermore, accuracy of electronic systems used for staffing levels and bed state were underused. There were gaps in information and furthermore information within the system was unreliable (together defined as 'defects'). These defects mandated bed managers' physical presence on each ward to obtain reliable data. This led to unwarranted critical care stays and resultant high rates (up to $40 \%$ ) of elective operation cancellations requiring a critical care bed.

The Leeds Improvement Method using principles of the Toyota Production System aimed to improve patient flow through critical care and to assess the impact on elective case activity. Problems were identified and changes were implemented over a 1-week period. The changes included measures to reduce time taken for collation of critical bedstate information and improving patient and staffing data quality collected in the electronic patient management system (EPMS) and electronic staffing record (ESR). Impact was monitored for 30 days pre-implementation and postimplementation.

Following intervention, the time taken by the bed manager to gather live bed-state information decreased from 50 to $9 \mathrm{~min}$; the EPMS storing correct bed-state data was improved from $71 \%$ to $0 \%$ defect; the ESR was improved from $100 \%$ to $4 \%$ defects; critical care patient step-downs occurring at night (after 20:00) improved from $80 \%$ to $20 \%$; and the number of cancelled elective cases over a 30-day period reduced from nine to one. Implementing these organisational efficiencies can significantly improve critical care patient flow and elective case throughput.

\section{PROBLEM}

Our institution, the Leeds General Infirmary, is a busy high-volume university teaching hospital and tertiary referral centre for neurosurgery. It serves an approximate regional catchment population of three million and performs over 3000 neurosurgical and spinal operations per year. There is a neurosurgical critical care bed capacity of 15 (7 intensive care beds and 8 high-dependency beds), in addition to 60 level 1 (ward level) beds. Despite this large bed base in neurosurgery, we have observed that there have been frequent and often significant delays to patient step-downs from critical care. The multidisciplinary team has also recognised the knock-on effects that this has caused in terms of frequent late cancellations (on the day) of surgical cases due to lack of identifiable postoperative critical care beds for these patients. The scale of this problem has been observed at rate of up to $40 \%$ of total case volume cancelled on a month-to-month basis due to lack of critical care beds.

The aim of this quality improvement project was to improve patient flow through neurosurgical critical care by reducing the time taken for the bed manager to collate critical bed-state information down to no more than $10 \mathrm{~min}$; achieving zero defects in the electronic patient management system (EPMS) and electronic staffing record (ESR); to increase the number of critical care early step-downs by decreasing the number of late critical care step-downs (occurring after 20:00) and reducing the extent of elective cancellations by at least $50 \%$.

\section{BACKGROUND}

It has been increasingly recognised that cancellations of elective surgical operations due to lack of bed capacity and poor inpatient flow is a significant problem. This is reflected in numerous literature reports with operation cancellation rates as high as $63 \%$ of surgical volume at some institutions. ${ }^{1-4}$ Factors cited that contribute to the often-poor critical care bed flow include case mix, staff shortages and 'bottle-neck' issues surrounding delayed patient repatriation. ${ }^{1}$ Regarding impact, having surgery has been shown to represent a significant life event for patients and their relatives, and cancelling it represents a major adverse influence on this. Furthermore, cancelled elective surgeries duplicate workload, diminish efficiency, waste operating room time and increase costs. ${ }^{5}$ 
The Leeds Improvement Method (LIM) is derived from the lean methodologies for efficient productivity of the Virginia Mason Production System (VMPS) ${ }^{6}$ and the Toyota Production System (TPS). ${ }^{7}$ The overarching principles are consistent across all the systems, although the methodology varies. These principles include removing waste, eliminating rework, levelling workload, standardising tasks, continuous improvement and decisions by consensus with rapid implementation. ${ }^{89}$ The success of TPS and VMPS are acknowledged to be related to the culture they create around the technical framework of lean rather than the tools they used. ${ }^{67}$ With regard to context, there is the size and structure of the organisations involved. Toyota is a large multinational company made up of smaller relatively homogeneous plants. Likewise, Virginia Mason, though a third the size of Leeds Teaching Hospitals Trust (LTHT), has similar functioning subunits and very few subspecialty teams. This contrasts with LTHT, which has both a large size and high complexity (due to multiple varied and heterogeneous functioning subspecialty departments and teams). This key attribute underlies the difference between LIM versus TPS and Virginia Mason methodologies. While TPS and Virginia Mason are centred around an organisation-wide 'all-in' approach to gathering data and making implementations/changes across multiple similar areas simultaneously, the size and subspecialty practice complexity of LIM mandates the gathering of data from discrete practice areas individually and the implementation of changes to discrete practice areas on an individual basis only, before considering wider replicability across the trust.

Patient flow through neurosurgical critical care was identified as such an example of a discrete practice area for improvement within LTHT. There are several examples in the literature of the use of lean principles in healthcare, and so the use of such a methodology to improve patient care is well documented and certainly not novel. ${ }^{10-13}$ However, the use of the LIM specifically to improve patient flow in the critical care environment (in this case for neurosurgical patients) has little published data behind it.

One of the fundamental elements of VMPS and TPS is genchi genbutsu, translated as 'real location, real thing', which essentially means 'go and see', and this is done on the genba (out in the work place, on the ground with the work force). Both VMPS and TPS would ensure that direct observation forms an essential part of their data collection and understanding of the 'current state' or 'point of departure' ahead of improvement efforts. This genba approach was adopted by the LIM. The genba approach was used in our project to understand the problems involved in critical care patient flow (preintervention) and enabled identification of several areas as targets for improvement, detailed further.

\section{Paper records of staffing levels}

These were not visible to bed managers and they only had access to the ESR. The electronic system for staffing was neglected and these 'defects' resulted in significant perceived inconsistencies in staffing levels and so were not easily incorporated into the analysis of patient flow.

\section{Underuse of the EPIMS}

At our institution, the EPMS contained information that was often very inaccurate. These defects resulted in significant inconsistencies in perceived bed state.

\section{Poor visibility of patient step-down status}

Critical care staff felt there was little visibility of critical care patients ready for step-down or to remain within their current acuity level.

\section{Poor communication between bed managers}

Both ward and critical care bed managers had no overlap between shifts to communicate bed-state issues. In the current state, they spent a large majority of their time walking between wards and discussing with the staff in person to collect the data they needed (bed state and staffing levels).

\section{Opaque role duties}

The current bed management involved use of handwritten notes, without clear structure, leading to variation in the format of forms used to collect information. This made the role of bed managing difficult to transition between shifts and for cover at short notice.

It has been shown that there are three main staffing choices for accommodating fluctuating demand on the health system: having enough staff at all times, below peak demand staff levels (whereby increased pressure is tolerated by the service) and dynamic staffing that reacts to demand. ${ }^{14}$ LTHT staffing can practice dynamic staffing, but this relies on a complete and up-to-date overview (ESR). Acquisition of the aforementioned information necessitated the physical presence of the bed managers on the wards, with attendant delays consuming important nursing time.

The combined result of all these factors led to frequent elective patient cancellations and significant delays in patient flow from critical care to ward-level beds. It has been demonstrated that early morning discharge helps to facilitate flow through critical care departments ${ }^{15}$ and therefore, a key target of our project in improving critical care patient flow was to increase the rate of morning discharges and accordingly reduce the rate of late discharges.

\section{MEASUREMENT}

This project involved three phases: initial observation of patient flow, critical care step-downs and elective case cancellations over a 30-day period; subsequent intervention of the LIM over 7 days; and postintervention observation over 30 days to assess the impact. Initial data collections were focused at observing during a standard working 30-day period to gain an overview of the problem. There were four areas that were identified that could be 
improved on and measured: the time taken for the critical care bed management team to collate information around the bed state, accuracy of the EPMS and ESR, number and time of patients stepping down from critical care and the number of cancelled elective patients.

Our baseline measurements of the current state determined that the critical care bed management team took $50 \mathrm{~min}$ to collate information around the bed state. Our electronic systems were remarkably underused with $71 \%$ defects within the EPMS and 100\% defects (no entries inputted) within the ESR. There were nine cancelled elective cases and $80 \%$ of patient step-downs occurred after 20:00 from critical care during the night shift.

Collecting patient flow information as early and quickly as possible enables more efficient planning of surgical operating lists. When combined with more patients stepping down earlier in the day, 'go or no go' decisions can be made for neurosurgical cases, especially those cases that require more than 4 hours for the afternoon operating slots. A goal of zero defects within the electronic systems allows robust and reliable information to deliver maximal efficiency.

\section{DESIGN}

The intervention development involved multiple stakeholders, including a matron, senior sister, registrar, foundation doctor and two bed managers (one each from the ward and critical care), enabling 'decisions by consensus'. This enabled 'rapid implementation' of potential highimpact areas of improvement over 7 days. There was no patient involvement in the design of this project.

Each member within the research team outlined the time taken for tasks to be performed within their area. Bed managers mapped out where they walked each day, how they obtained the staffing and bed state and the time taken. Nursing staff expressed their frustrations of the little visibility of staffing for the critical care department and how handwritten notes were being made in a book which could sometimes be illegible. The department required a minimum number of staff to function at full capacity and beds would need to be closed if there were not enough nurses to staff them.

The following interventions were decided on:

1. Moving staffing from paper records to an electronic system. Training would be delivered to the senior nursing staff in use of the new electronic system. Changes to the electronic system would be limited to the senior nursing staff. A digital champion would be named who would be able to troubleshoot issues. Data entered into the staffing record would be checked regularly for accuracy and all shift swaps or absences would be recorded. A manual will be created for future reference.

2. Real-time, accurate information updates to the EPMS. Training would be delivered to all nursing staff and ward clerks on the use of the specific features within the EPMS to enable adding and moving patients within the system. Patients would be added to the EPMS as a priority on admission, placed within the correct bed space and location updated as they were moved. Data entered into the EPMS would be checked regularly for accuracy. A manual will be created for future reference.

3. Step-down status flag. Development of a system to improve patient step-down status by adding a flag to the EPMS by the nurse in charge (NIC) on the night shift. This would be reviewed and confirmed by the medical team on the morning ward round.

4. Time to communicate issues. Once interventions 1 and 2 were achieved, the bed managers will be able to remotely view the ESR and EPMS for bed allocation state rapidly. Protected time will be created to communicate identified issues and to problem solve rather than data collect.

5. Standardising role documentation. Duties and responsibilities will be outlined by the members of the bed management team and a full manual will be created for future reference.

The interventions mentioned earlier targeted key areas for improvement to achieve the following endpoints:

1. No use of paper staffing records and zero defects in the ESR.

2. Elimination of defects within the EPMS.

3. Visible step-down status of patients within critical care.

4. Reduction in the time taken by bed managers to collate bed and staffing state.

5. Easier staff shift transitions.

Together the aforementioned data aimed to provide a 'live' and dynamic view of patient flow and capacity to the bed manager prior to the daily hospital operation meetings (morning, afternoon and evening). This aimed to deliver better visibility and planning of valuable resources. The intervention would free up nurses for clinical duties achieving the principle of 'removing waste', reduce the amount of time to obtain the desired information, and information could be accessed remotely from anywhere in the hospital. These areas would be specifically targeted with several 'plan, do, study, act' (PDSA) cycles.

\section{STRATEGY}

The LIM $^{16}$ was developed after working closely with the Virginia Mason, ${ }^{17}$ which is regarded as one of the most efficient healthcare providers in the world. They have achieved these goals by applying the 'lean' principles used in the TPS, which are removing waste, eliminating rework, levelling workload, standardising tasks, continuous improvement and decisions by consensus with rapid implementation. ${ }^{89}$ 


\section{PDSA cycle 1}

The two main areas that were felt to have the biggest impact on the flow of patients through the critical care department were staffing and bed state and therefore were targeted first (interventions 1 and 2). There was little visibility, which made planning capacity difficult. The aim was to have real-time updates to the EPMS and staffing record instead of the use of paper records. Updates would be carried out by the NIC overnight, so that information was readily available for the bed managers in the morning. This in turn would speed up patient flow through critical care by reducing the lead time for ascertaining the current bed state and staffing levels ready for the morning hospital daily operation meeting.

At the start of the project, several nurses were identified as digital champions and were given one-to-one training by members of the research team. Four sessions were then delivered to capture everyone who was required to make changes to either the ESR or the EPMS and teach them on how to use these systems. Contact details for the digital champions or research team were then made available for future troubleshooting. Both the EPMS and ESR on the first day of the project were checked for accuracy and the paper records were abolished.

\section{PDSA cycle 2}

It was felt that now there was a better visibility of staffing and bed state, the NIC on the night shift could identify and highlight patients who were likely to be stepped down (intervention 3). The flow would be from either intensive care to the high-dependency unit or from the highdependency unit to a ward-level bed. During every night shift, potential patients for step-down were highlighted on the EPMS. This expedited the final step-down decisions on the morning ward rounds by the medical team to 'level out the workload'. The morning ward round could focus on these patients early and if they were confirmed to be medically safe, allowed to flow downstream. Furthermore, any issues that were holding up these patients could be addressed in a timely fashion.

\section{PDSA cycle 3}

As the project started to improve flow though the neurosurgical critical care department, the workload on the nursing staff was relieved as information did not have to be presented to the bed management team in person. The bed managers could also quickly identify patients ready for step-down to the ward (intervention 4). Unexpectedly, time was freed up for not only the bed managers but also for the nurses. It was hypothesised that a quick meeting or 'huddle' between the charge nurses and bed managers (for both the ward and critical care departments) would be beneficial. The aim of this meeting was to further highlight specific needs for potential step-down patients to the receiving team, important events during their stay and improved patient safety. It was thought that this would make the transition smoother for the patient performing this ahead of time. The current standard operating procedure was to hand this information over between teams on the day of step-down. These changes highlight the 'continuous improvement' shown in this project.

\section{PDSA cycle 4}

The time for the huddle was changed as it was found to interfere with other activities during the day. The time was changed from 16:00 to the earlier time of 10:00 and was rapidly implemented. This was demonstrated to have better attendance and worked better to prepare the staff on the day shift for step-downs.

\section{PDSA cycle 5}

As the project was gaining validity from improving flow through the critical care department, manuals needed to be created (intervention 5). The key members for each team (medical, nursing and bed managers) ensured all forms were categorised and descriptions of essential procedures for databases were added. This 'standardisation of tasks' allowed any staff member to cover this role with minimal support. It was tested within the wider team to ensure that the tasks which needed to be carried out could be completed without outside help and only from the standard work description. Fine adjustments were made to these to ensure that there was no ambiguity.

\section{RESULTS}

The mean time taken by the bed manager to gather live bed-state information ready for the daily hospital operations meeting was reduced from 50 min preintervention to 9 min following the intervention $(\mathrm{p}<0.01$, paired Student t-test).

The EPMS, which identifies correct patient bed and ward allocation data, was improved from $71 \%$ defects (incorrect or incomplete data) to $0 \%$ defect following the intervention $\left(\mathrm{p}<0.01, \chi^{2}\right.$ test $)$.

The ESR was improved from $100 \%$ defects (no entries inputted) to $4 \%$ defects following the intervention $\left(\mathrm{p}<0.01, \chi^{2}\right.$ test $)$.

Rate of delayed critical care patient step-downs after 20:00 improved from $80 \%$ (40 patients out of 50 ) to $20 \%$ (10 patients out of 50$) \quad\left(p<0.01, \chi^{2}\right.$ test $)$. There was an increase in the number of elective bed allocations identified by the daily 8:00 operation meeting (figure 1). The number of cancelled elective cases was reduced from nine cases over 30 days to one case over 30 days postintervention (figure 2).

Audit of attendance of the huddle was performed, and it was initially very well attended. The huddle consisted of five members, which included the NIC of critical care, the NIC of the male ward, the NIC of the female ward, the wards' bed manager and the critical care bed manager. Initially, $74 \%$ of the meetings ( 17 of a possible 23 ) went ahead, which fell to $65 \%$ (20 of a possible 31 ). Qualitative feedback from the staff demonstrated this decline was shown be due to clashes with afternoon activities. Nevertheless, all staff surveyed on a Likert scale (range: 


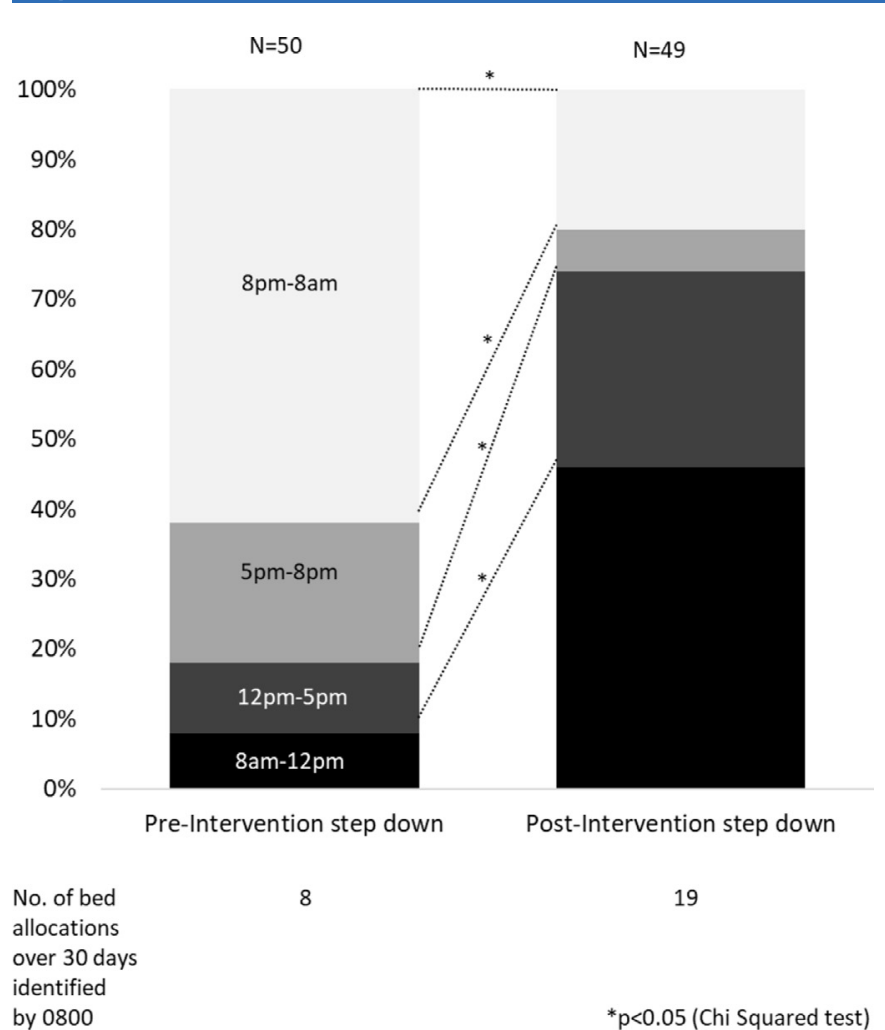

Figure 1 Rate of delayed critical care patient step-downs by time and the number of elective bed allocations over a period of 30 days pre and post intervention

very satisfied, satisfied, neither, dissatisfied, very dissatisfied) confirmed that they were 'very satisfied' with having a huddle. Subsequently, the huddle was moved to the morning and was much better attended and sustained with $85 \%$ attendance (23 of a possible 27). Other comments from the qualitative feedback included the nurses feeling they could 'better plan for patient step-downs', 'prioritise bed space cleans and moves' and 'highlight the urgency of electronic discharge notes to the medics to free up beds for the critical care step-downs'.

It was noted that between the two periods studied, there were no changes in other factors such as staff shortages, theatre closures or procedures performed.

\section{Lessons and limitations}

The aim of this quality improvement project was to improve patient flow through neurosurgical critical care using a sustainable approach that could be replicable in other specialised critical care units throughout LTHT. To enable this, the project focused on reducing the time taken for the bed manager to collate critical bed-state information, achieving better use of the EPMS and ESR, increasing the number of early critical care step-downs and reducing the number of late critical care step-downs, and overall reducing the extent of elective surgery cancellations. The project followed several PDSA cycles representing continuous improvement that were instrumental in making the project agile to respond to developments as the project unfolded and in delivering changes that ultimately achieved patient flow improvements. Therefore, the importance of the PDSA cycles was a key lesson from the project.

The interventions involved multiple stakeholders including a matron, senior sister, registrar, foundation doctor and two bed managers (one each from the ward and critical care) enabling decisions by consensus. A significant lesson from this multidisciplinary approach was the huge importance of having key people 'buy in' at an early stage to drive the project forward to completion. This enabled sharing of the wealth of experience from these individuals that worked day-to-day delivering patient care, shared identification of the key issues and rapid implementation of changes. During the roll out stage, hindsight suggests that it might have helped to have had more senior nurses involved at the outset (as opposed to just one) because on days when the senior sister was not on shift, there were questions and issues about implementation-related tasks that would have been more quickly dealt with before the critical morning rounds had there been another or a greater pool of senior nurses familiar and involved in the project. The initial teaching sessions and contact details for the research team mitigated some of these issues, but not all could be resolved before the next morning.

We recognise that there is always a certain amount of resistance to change and departure from the 'usual way

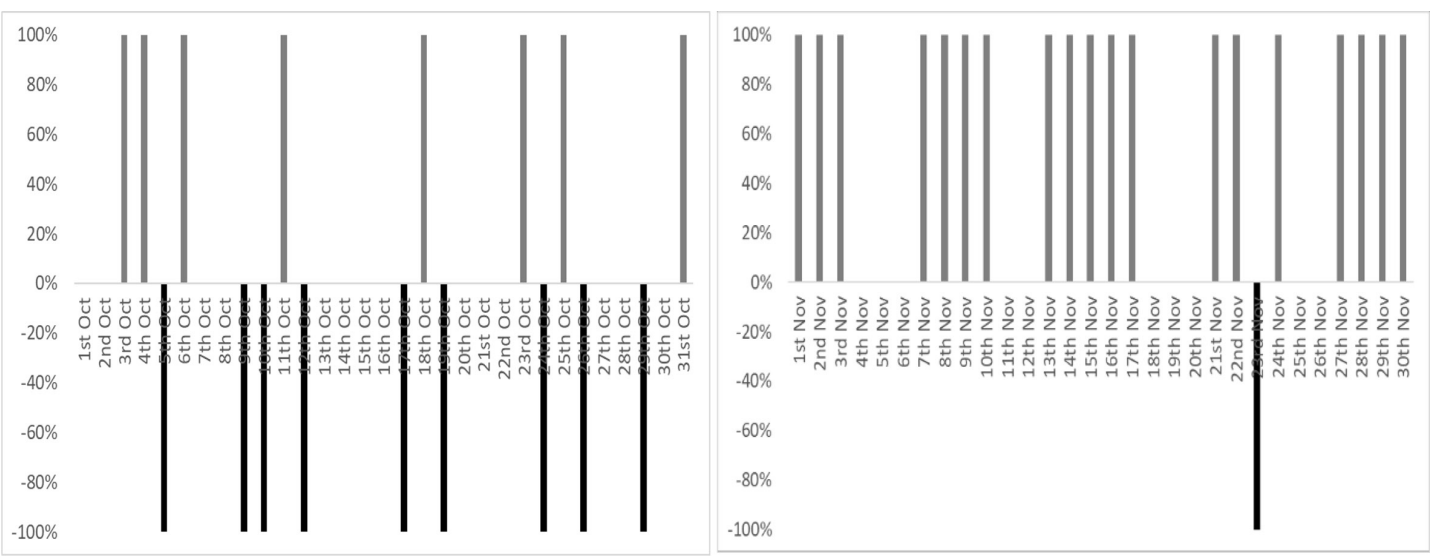

Figure 2 Elective case throughput over a period of 30 days pre and post intervention 
things are done', and this project team faced the same problem, particularly in view of the fact that the staff involved were for the most part long-term, non-rotating workers. However, as staff could see that the changes were positive (step-down activity was announced at daily nursing staff handover meetings) and as the nursing staff felt they had freed up more clinical time to spend with patients (less time occupied with multiple staffing and bed-state discussions), the new implementations systems were increasingly adopted by all.

While we have seen a significant positive effect of our interventions on critical care patient flow and elective case cancellations, it is possible that these effects occurred due to variation in natural patient flow rather than our interventions, and we acknowledge that our project is based on observations on a limited sample size and short observation period. Nevertheless, with our recorded data, we were able to perform statistical tests that showed significant results, although ideally univariate or multivariate analyses would have further strengthened our data and conclusions.

The improved use of the EPMS and ESR substantially improved the flow of patients through the critical care department and reduced the number of cancelled elective cases. For continued success, a potential future development is to the EPMS system itself to include a more granular/stratified approach to a patient's step-down status, for example, employing a 'traffic light' grading system from 'green' (ready to step-down), 'amber' (few key outstanding issues that are near resolving), to 'red' (needs to remain in critical care). This may allow focus on amber patients to get them ready to be stepped down (in addition to green patients) or more rapid consideration of moving amber patients when dealing with emergency scenarios that require immediate patient flow. This traffic light system is currently being explored as a future expansion of our project.

\section{CONCLUSION}

While there are examples abundant in the literature of lean principles applied within healthcare, to the best of our knowledge, this report is the first to show a significant impact of lean principles using the nuanced LIM in improving neurosurgical critical care flow and reducing elective case cancellations, when properly implemented. Furthermore, the daily multiple stakeholder involvement helped build relationships between critical care and the downstream ward teams who felt better prepared when receiving patients. The improvements to the bed manager's standard work description, including databases and standardised tasks, facilitated development opportunities for unfamiliar staff to easily take on the bed manager role and provided consistency to the service provided. Considering the significant success of the interventions, these changes have now become incorporated into routine practice within the neurosurgery department. However, it is important to share our improvement methodologies with the wider trust to enable opportunities to improve patient flow in other surgical departments, and this is currently the subject of further clinical governance work in our trust.
Acknowledgements We acknowledge the contributions and hard work of the Leeds Teaching Hospital Trust neurosurgical critical care nursing staff with special thanks to Matron Steve King; Janet Campbell and Joanne Clapham the bed management team; Jimmy Parvin and Dawn Horanszkey, the Leeds Improvement Method team; and finally, Dr Yvette Oade, chief medical officer and Julian Hartley, chief executive officer; without their support this project would not have been possible.

Contributors JM, SM and AS participated sufficiently in the writing of the paper to take public responsibility for all the information submitted within this article. All authors reviewed the final version of the article and approved it for publication. There are no prior publications or submissions with any overlapping information, including studies and patients. The authors declare no conflicts of interest in relation to the writing of this article.

Funding The authors have not declared a specific grant for this research from any funding agency in the public, commercial or not-for-profit sectors.

Competing interests None declared.

Patient and public involvement Patients and/or the public were not involved in the design, or conduct, or reporting, or dissemination plans of this research.

Patient consent for publication Not required.

Provenance and peer review Not commissioned; externally peer reviewed.

Data availability statement All data relevant to the study are included in the article.

Open access This is an open access article distributed in accordance with the Creative Commons Attribution Non Commercial (CC BY-NC 4.0) license, which permits others to distribute, remix, adapt, build upon this work non-commercially, and license their derivative works on different terms, provided the original work is properly cited, appropriate credit is given, any changes made indicated, and the use is non-commercial. See: http://creativecommons.org/licenses/by-nc/4.0/.

ORCID iD

James Meacock http://orcid.org/0000-0002-2775-1838

\section{REFERENCES}

1 Robb WB, O'Sullivan MJ, Brannigan AE, et al. Are elective surgical operations cancelled due to increasing medical admissions? Ir J Med Sci 2004;173:129-32.

2 Dimitriadis PA, lyer S, Evgeniou E. The challenge of cancellations on the day of surgery. Int J Surg 2013;11:1126-30.

3 Herrod PJJ, Adiamah A, Boyd-Carson H, et al. Winter cancellations of elective surgical procedures in the UK: a questionnaire survey of patients on the economic and psychological impact. BMJ Open 2019;9:e028753.

4 Sahraoui A, Elarref M. Bed crisis and elective surgery late cancellations: an approach using the theory of constraints. Qatar Med J 2014;2014:1.

5 Gillies MA, Wijeysundera DN, Harrison EM. Counting the cost of cancelled surgery: a system wide approach is needed. $\mathrm{Br} J$ Anaesth 2018;121:691-4.

6 VMPS. Virginia Mason production system (VMPS. Seattle: Virginia Mason, 2020. https://www.virginiamason.org/vmps

7 Toyota Motor Corporation. Toyota Production System | Vision \& Philosophy | Company. Toyota Mot. Corp. Off. Glob. Website, 2020. Available: https://global.toyota/en/company/vision-and-philosophy/ production-system/index.html [Accessed 1 Aug 2020].

8 Burgess N, Radnor Z. Service improvement in the English National health service: complexities and tensions. Journal of Management \& Organization 2012;18:594-607.

9 Parkhi SS. Lean management practices in healthcare sector: a literature review. BIJ 2019;26:1275-89.

10 Spagnol GS, Min LL, Newbold D. Lean principles in healthcare: an overview of challenges and improvements. IFAC Proceedings Volumes 2013;46:229-34.

11 Catalyst N. What is lean healthcare? NEJM Catal https://catalyst. nejm.org/doi/abs/

12 Andersen LL, Jørgensen MB, Blangsted AK, et al. A randomized controlled intervention trial to relieve and prevent neck/shoulder pain. Med Sci Sports Exerc 2008;40:983-90.

13 Antony J, Kumar M. Lean and six sigma methodologies in NHS Scotland: an empirical study and directions for future research. QIP Journal 2012;16.

14 Litvak E, Fineberg HV. Smoothing the way to high quality, safety, and economy. N Engl J Med 2013;369:1581-3. 
15 Hostetter M, Klein S. In focus: improving patient Flow - In and out of hospitals and beyond | Commonwealth fund, 2020. Available: https:// www.commonwealthfund.org/publications/newsletter-article/focusimproving-patient-flow-and-out-hospitals-and-beyond [Accessed 1 Aug 2020].
16 Leeds Teaching Hospital Trust. The Leeds improvement method, 2019. Available: https://www.leedsth.nhs.uk/about-us/the-leeds-way/ the-leeds-improvement-method/ [Accessed 23 Jun 2020].

17 Burgess N, Radnor Z. Evaluating lean in healthcare. Int J Health Care Qual Assur 2013;26:220-35. 